\title{
Passive Intermodulation Due to Self-Heating in Printed Transmission Lines
}

\author{
Eduard Rocas, Student Member, IEEE, Carlos Collado, Senior Member, IEEE, \\ Nathan D. Orloff, Jordi Mateu, Senior Member, IEEE, Alberto Padilla, \\ Juan M. O'Callaghan, Senior Member, IEEE, and James C. Booth
}

\begin{abstract}
This paper proposes a mechanism by which third-order intermodulation distortion, due to self-heating, is generated in transmission lines. This work shows how transmission lines made of several materials, whose properties are independent of the electric and magnetic fields, can generate important levels of intermodulation distortion. A circuit model supported by finite-element simulations is presented to account for the temperature generation and also for its impact on the nonlinear performance. Closed-form expressions are used to calculate the generated intermodulation products and are derived from the circuit model and compared with simulations. Finally, measurements and simulations of different transmission lines are presented, showing very good agreement.
\end{abstract}

Index Terms-Intermodulation distortion, nonlinearities, printed lines, self-heating, temperature, thermal effects, transmission lines.

\section{INTRODUCTION}

I NTERMODULATION distortion and harmonic generation in passive transmission lines are usually associated with the dependence of the material properties on the electromagnetic fields. Such is the case for transmission lines made of superconductors [1] or ferroelectric materials [2]. However, several papers have shown the existence of third-order intermodulation distortion, even in transmission lines made of inherently linear materials, such as copper on commercial substrates [3], [4]. In this case, and depending on the speed of the signal modulation, self-heating mechanisms can be the cause of third-order intermodulation distortion, which can generate serious problems in

Manuscript received September 15, 2010; accepted October 08, 2010. Date of publication November 29, 2010; date of current version February 16, 2011. This work was supported in part by the U.S. Government and by the Spanish Ministry of Science and Innovation under Grant TEC-2009-13897-C03-01/TCM. The work of E. Rocas was supported by the Spanish Ministry of Education under a Ph.D. Fellowship (BES-2007-16775). The work of C. Collado was supported by the Generalitat de Catalunya under a grant (2008-BE2-00196). The work of A. Padilla was supported under a Ph.D. Fellowship (AP2008-02235). This paper is an expanded paper from the IEEE MTT-S Int. Microwave Symposium, Anaheim, CA, May 23-28, 2010.

E. Rocas and C. Collado are with the Department of Signal Theory and Communications, Universitat Politècnica de Catalunya (UPC), Barcelona 08034, Spain, and are also with the National Institute of Standards and Technology (NIST), Boulder, CO 80305 USA (e-mail: eduard.rocas@tsc.upc.edu).

N. D. Orloff and J. C. Booth are with the National Institute of Standards and Technology (NIST), Boulder, CO 80305 USA.

J. Mateu, A. Padilla, and J. M. O'Callaghan are with the Department of Signal Theory and Communications, Universitat Politècnica de Catalunya (UPC), Barcelona 08034, Spain.

Color versions of one or more of the figures in this paper are available online at http://ieeexplore.ieee.org.

Digital Object Identifier 10.1109/TMTT.2010.2090356 communication systems [5]. This occurs because temperature oscillations cause variations of the material properties without generating harmonics.

The dependence of device performance on temperature has always been a source of concern in the process of designing microwave devices exposed to temperature variations [6], [7] and the correct choice of materials plays a vital role. Attention has usually focused on minimizing the temperature dependence of material properties, as well as on minimizing the loss that is responsible for the temperature rise. Significant temperature dependence of material properties can cause the performance of the device to change considerably over a given temperature range and can also generate nonlinear distortion.

While thermally generated intermodulation distortion is a well-known nonlinear process in power amplifiers where thermal effects have always been a matter of concern [8]-[10], only few papers have been published on this topic. This has happened despite the impact that temperature variations can have on the linear and nonlinear performance of passive microwave devices. The effect of resistive heating on intermodulation distortion generation in electrical contacts is analyzed in detail in [11]. More recently, [12] has shed light by modeling this effect in lumped devices such as attenuators, microwave chip terminations, and coaxial terminations.

Fewer papers have focused on the self-heating process due to the lossy nature of distributed structures, such as transmission lines [13], [14]. The approach found in the $3 \omega$-method [15] is a rigorous procedure to extract thermal material properties by measuring the third-harmonic generated by self-heating in metallic strips at audio frequencies. The existing nonlinear transmission line models [16] do not address these underlying mechanisms of passive intermodulation generation in printed transmission lines and are based strictly on phenomenological approaches. Only [17] and [18] analyze the thermal heating contribution to intermodulation in coaxial waveguides and transmission lines, respectively.

This work presents a circuit model to account for the temperature rise due to self-heating in transmission lines and describes in detail how these temperature variations generate third-order intermodulation distortion. The distributed electromagnetic circuit model of a transmission line is coupled to a thermal domain that models the heat generation and propagation, yielding the temperature at each point of the line. The temperature rise is then used to change the material properties on the electromagnetic domain circuit model. We extend the results in [19] by presenting finite-element thermal simulations to support the model. In addition, we validate the model with measurements 


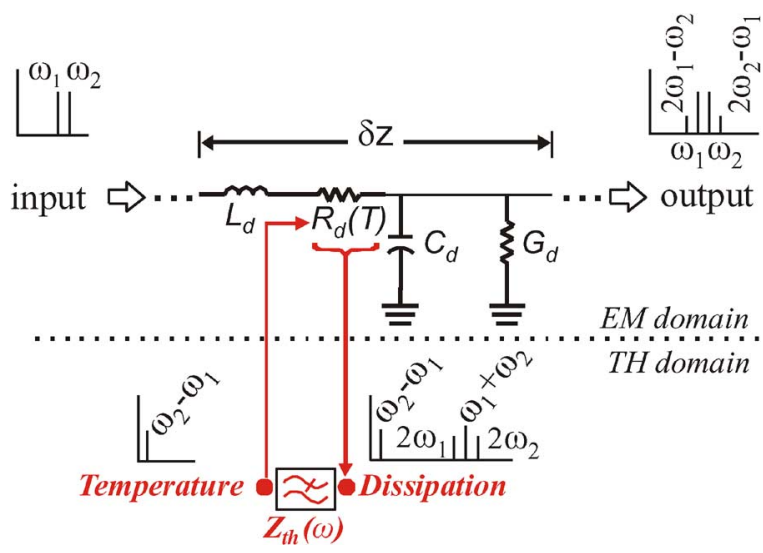

Fig. 1. Passive intermodulation distortion generation process in a transmission line segment of length $\delta z$. Resistive losses are responsible for temperature fluctuations that, in turn, change the metal resistivity.

of coplanar waveguides on single crystal sapphire substrates, made with conductors of three different metals in order to give a better understanding of the intermodulation generation mechanism. The assumption of weak distributed nonlinearities is made so that the nonlinearities represent a small perturbation of the linear performance.

Closed-form expressions that allow prediction of intermodulation levels on matched transmission lines are derived from the circuit model implementation and compared with circuit simulations. Finally, the model is tested with intermodulation measurements on lines of several lengths fabricated from different conductor materials with good agreement between modeled and measured results.

\section{Mechanism of PASSIVE InTERMOdUlation GENERATION DUE TO SELF-HEATING IN DISTRIBUTED TRANSMISSION LINES}

The scheme in Fig. 1, in which the two-tone test is reproduced, shows the generation process of third-order intermodulation distortion due to self-heating in a transmission line along the $z$-axis. The input signals, $\omega_{1}$ and $\omega_{2}$, can also be understood as an AM modulated signal with carrier suppression centered at $\omega_{0}$ with an envelope frequency $\Delta \omega / 2$ so that $\omega_{1}=\omega_{0}-\Delta \omega / 2$ and $\omega_{2}=\omega_{0}+\Delta \omega / 2$.

If a low-loss substrate is considered, the loss due to the conductor dominates and the dissipated power $Q_{d}(z, \omega)$ in each elemental segment $\partial z$ of transmission line is proportional to the square of the current through that segment. This results in dissipated power, with frequency components at dc, $\Delta \omega, 2 \omega_{1}, 2 \omega_{2}$, and $\omega_{1}+\omega_{2}$. Each of the frequency components of the dissipated power gives rise to temperature oscillations $T$, according to the corresponding thermal impedance $Z_{\mathrm{th}}(\omega)$ at each specific frequency [9]

$$
T(z, \omega)=Z_{\mathrm{th}}(\omega) Q_{d}(z, \omega)
$$

As a result, the steady-state temperature rise changes the material properties and, as a result, the linear performance of the device, as well. On the other hand, the oscillating temperature generates third-order intermodulation distortion. The slow dynamics related with the heat propagation in a media translates into a low-pass-filter behavior of the thermal impedance [20], thus making the dissipated signal at $\Delta \omega$ the most relevant oscillating temperature component. As in the steady-state case, temperature variations at $\Delta \omega$ can change materials properties; for example, the metal resistivity, resulting in third-order intermodulation distortion at $2 \omega_{1}-\omega_{2}$ and $2 \omega_{2}-\omega_{1}$. In this work, a temperature-independent dielectric is considered. A detailed scheme of the generation process in an infinitesimal section of transmission line can be seen in Fig. 1.

As a result of the above-described process, measurements of the third-order intermodulation signals at different separations between tones might be used to unveil the low-pass filter shape of the thermal impedance [10] since

$$
P_{3 \mathrm{IMD}} \propto\left|Z_{\mathrm{th}}(\Delta \omega)\right|^{2}
$$

where $P_{3 \text { IMD }}$ is the third-order intermodulation distortion power.

\section{SElf-Heating Mechanism Model}

A circuit model of a transmission line, based on the previously described mechanism, could be used for the analysis and simulation of nonlinear effects under any type of input signal. Further, it could also be used, for example, to predict nonlinearities in more complex devices that make use of these transmission lines, such as filters [21]. The challenge of such a model, however, is based on the interaction of two physical domains, the electromagnetic and thermal domains, both of which must be properly modeled.

\section{A. Electromagnetic Domain}

A distributed implementation of the electromagnetic domain of a transmission line allows for relating the measured observables with the material properties [1]. The transmission line is constructed as a cascade of cells. Each cell contains the distributed parameters modeling a segment of a transmission line $R_{d}(T), L_{d}, C_{d}$, and $G_{d}$. These stand for the temperature-dependent distributed resistance and the distributed inductance, capacitance, and conductance, respectively [22]. The resulting nonlinear telegrapher equations are

$$
\begin{aligned}
& \frac{\partial v(z, t)}{\partial z}=-R_{d}(T) i(z, t)-L_{d} \frac{\partial i(z, t)}{\partial t} \\
& \frac{\partial i(z, t)}{\partial z}=-G_{d} v(z, t)-C_{d} \frac{\partial v(z, t)}{\partial t} .
\end{aligned}
$$

The distributed resistance $R_{d}(T)$ has contributions from each of the conductors in the transmission line, which act as heat generators. This allows us to simulate the temperature rise at each conductor independently and also to simulate the contribution to the intermodulation arising from each conductor. In a coplanar waveguide, the center conductor resistance $R_{d, N}(T)$ and the ground planes resistance $R_{d, M}(T)$ can be considered separately, as shown in Fig. 2

If a low-loss nondispersive substrate is used, $G_{d}$ is negligible and $C_{d}$ is constant over frequency. On the other hand, $R_{d, X}(T)$ and $L_{d}$ are frequency-dependent and need to be properly described at each frequency point.

Each contribution to the distributed resistance $R_{d, N}(T)$ and $R_{d, M}(T)$ can be expanded by use of a first-order Taylor's series, 


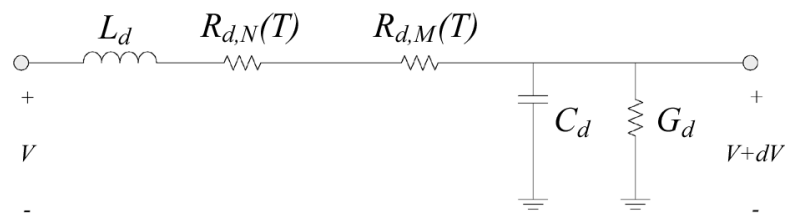

Fig. 2. Electromagnetic-domain model of an infinitesimal section of a transmission line.

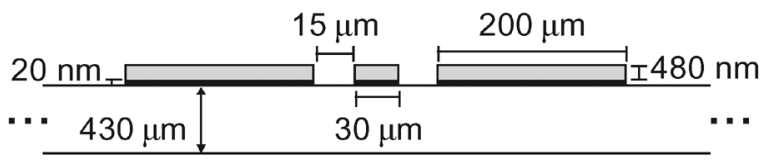

Fig. 3. Dimensions of the coplanar waveguides used in this work.

and casting it into a temperature-invariant term and a temperature-dependent term

$$
R_{d, X}(T)=R_{d, X, T_{R}}+\Delta R_{d, X} T
$$

where $X=N$ or $M$ stand for the center conductor and ground planes, respectively, and $R_{d, X, T_{R}}$ set the distributed resistance at room temperature $T_{R}$. The relation between the parameter $\Delta R_{d, X}$ and the temperature-dependent resistivity of the conductors is detailed in Appendix I.

\section{B. Thermal Domain}

A thermal model implementation of a transmission line should be able to predict the steady-state temperature rise and temperature oscillations that lead to intermodulation generation. Moreover, a physical relation with the material thermal properties and device geometry is necessary to allow the model to predict the nonlinear distortion of a device in the design stage.

1) Heat Generation in a Transmission Line: The geometry of a regular coplanar waveguide consists of two ground planes on each side of a center conductor. The metal layer varies, generally, from tens to thousands of nanometers thick and is deposited on a low-loss substrate. In such a structure, the generated heat in the metal strip is mostly dissipated by conduction through the substrate. This is because of its low thermal resistance to heat flow when compared to radiation and convection effects [20]. Fig. 3 shows the dimensions of the coplanar waveguides used in this work.

The first step toward the construction of a thermal model of a coplanar waveguide is to identify the heat sources. As previously mentioned, this work considers negligible dielectric losses in the substrate so that metal resistivity is the only source of dissipation. Fig. 4 illustrates the simulated current distribution at the beginning of the line $z=0$ of a coplanar waveguide, of the same dimensions as in Fig. 3, and made of gold on a sapphire substrate with a thin titanium adhesion layer. The transmission line is designed, considering dispersion, to be roughly matched at $6 \mathrm{GHz}$ by use of an electric resistivity of $\rho_{e}=27.67 \mathrm{n} \Omega \cdot \mathrm{m}$. The line is driven by two tones of $20 \mathrm{dBm}$ each, where the upper tone is set at $6 \mathrm{GHz}$ and the lower is $1 \mathrm{kHz}$ apart.

In the simulations of Fig. 4, the current density peaks are at the edges of the center conductor and at the inner edges of the ground planes. This has a direct translation in the location of

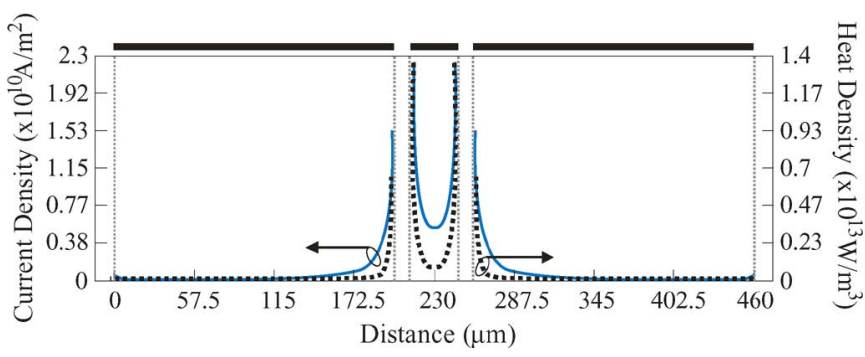

Fig. 4. Current distribution and joule heat at the beginning of a coplanar waveguide, driven by two tones at $20 \mathrm{dBm}$.

heat sources since the heat generated per unit volume can be expressed as [12]

$$
Q=J^{2} \rho_{e}
$$

where $J$ is the current density in $\mathrm{A} \cdot \mathrm{m}^{-2}$ and $\rho_{e}$ is the electric resistivity in $\Omega \cdot \mathrm{m}$. Fig. 4 also illustrates the heat density, obtained by use of finite-element simulations [23], due to (6).

The heat density surface integral on the center conductor results in an average dissipated power of $9.26 \mathrm{~W} \cdot \mathrm{m}^{-1}$ versus $3.54 \mathrm{~W} \cdot \mathrm{m}^{-1}$ for the ground planes, which clearly illustrates the fact that the center conductor contributes more to the overall dissipation. This gives an idea of the correspondent contribution of each metal strip to the distributed resistance, which follows the relation

$$
R_{d, X, T_{R}}=\frac{P_{d, X}}{I_{\mathrm{rms}}^{2}}
$$

where $P_{d, X}$ and $I_{\text {rms }}$ are the average dissipated power per unit length and the root mean square (rms) current, respectively.

For the coplanar waveguide under study, we used finite-element software [23] to get the distributed resistance contributions arising from the center conductor $R_{d, N, T_{R}}=2281 \Omega / \mathrm{m}$ and the ground planes $R_{d, M, T_{R}}=882 \Omega / \mathrm{m}$ at $6 \mathrm{GHz}$ and at room temperature. Therefore, the center conductor is expected to generate a higher temperature contribution, given its higher losses.

2) Steady-State Temperature Rise: Temperature rise, as a result of heat generation, follows a low-pass filter behavior, and thus is frequency dependent. Therefore, a clear idea of the temperature profile on the cross section of the coplanar waveguide is necessary to propose a thermal model implementation. Fig. 5 shows the steady-state temperature on the cross section of the previously presented coplanar waveguide, obtained with finiteelement simulation [23]. Radiation and convection effects were shown to be negligible through simulations. Table I contains the material properties used for the finite-element simulations utilized in this work.

The electric resistivity values are obtained by measurements of the transmission lines, explained in Section V. Other properties for $\mathrm{Au}, \mathrm{Pt}$, and Ti can be found in [24]. Sapphire properties are found in [25] and PdAu density and thermal conductivity is found in [26]. The specific heat value of PdAu is inferred from the Pd and Au specific heat since specific heat values for these metals are similar.

As shown in Fig. 5, the temperature distribution across the center conductor can be considered uniform, as it changes by less than 3\% from the edge to the center of the strip. The ground 


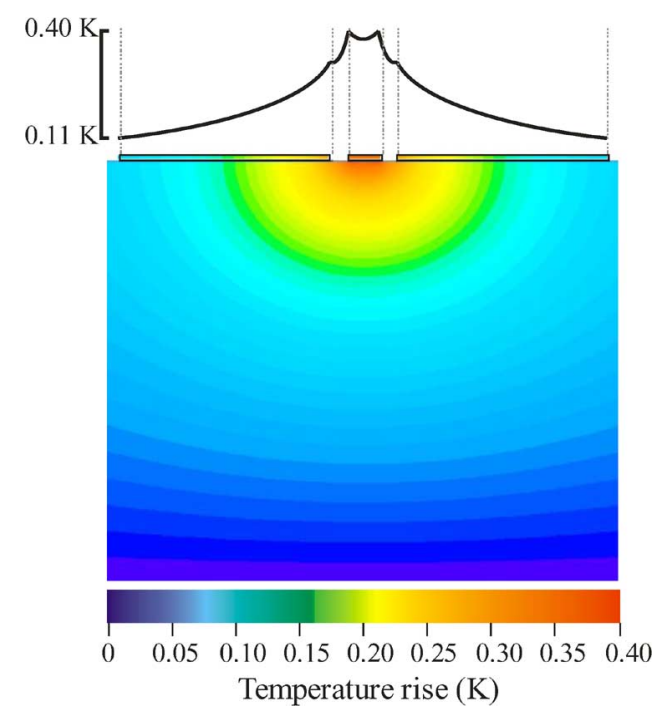

Fig. 5. Steady-state temperature rise profile on the cross section of the coplanar waveguide.

TABLE I

Material PRoperties Used IN THE Finite-Element Simulations

\begin{tabular}{lccccc}
\hline \hline & $\mathrm{Au}$ & $\mathrm{Pt}$ & $\mathrm{PdAu}$ & $\mathrm{Ti}$ & Sapphire \\
\hline Resistivity, $\rho_{e}(\mathrm{n} \Omega \cdot \mathrm{m})$ & 27.67 & 238.9 & 444.18 & 450 & - \\
\hline $\begin{array}{l}\text { Thermal conductivity, } \\
k\left(\mathrm{~W} \cdot \mathrm{m}^{-1} \cdot \mathrm{K}^{-1}\right)\end{array}$ & 301 & 69.1 & 26.1 & 17 & 42 \\
\hline $\begin{array}{l}\text { Specific heat, } c_{p} \\
\left(\mathrm{~J} \cdot \mathrm{kg}^{-1} \cdot \mathrm{K}^{-1}\right)\end{array}$ & 128 & 134 & 190 & 528 & 750 \\
\hline Density, $\eta\left(\mathrm{kg} \cdot \mathrm{m}^{-3}\right)$ & 19320 & 21450 & 12023 & 4500 & 3970 \\
\hline \hline
\end{tabular}

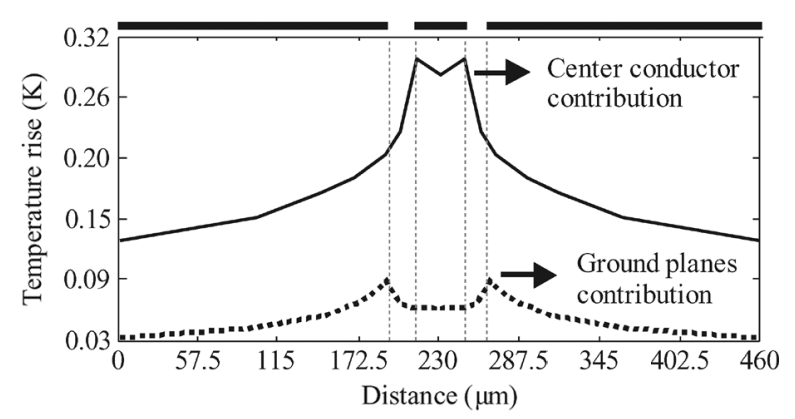

Fig. 6. Steady-state temperature contributions arising from the dissipation in the center conductor and the ground planes, independently.

planes, though, show a clear temperature gradient. Thus, additional finite-element simulations, by use of specifically implemented software, ${ }^{1}$ have unveiled the temperature-rise contributions arising from the center conductor and the ground planes independently. Fig. 6 shows the results of the simulation, where the ground planes contribute less to the overall temperature rise than the center conductor. A reasonable approximation of the temperature rise in the center conductor can, therefore, be obtained by considering only its dissipation.

3) Oscillating Temperature: The thermal penetration depth is defined as $\delta_{p}=4 \sqrt{k / c_{p} \eta \omega}$, which illustrates the fact that the resistance to heat propagation through materials depends not only on the material properties, but also the frequency [20]. In

\footnotetext{
${ }^{1}$ Specifically implemented 3-D electro-thermal finite-element software.
}

the previous equation, $k$ is the thermal conductivity, $\eta$ is the density, $c_{p}$ is the specific heat, and $\omega$ is the frequency of the temperature oscillations $\omega=\Delta \omega$. This translates into locally accentuated temperature distributions at higher frequencies with smaller maximum values. Fig. 7 shows the oscillating temperature profile at the surface of the cross section of the coplanar waveguide for different envelope frequencies, obtained by means of simulations.

Simulations reveal that the temperature rise at the center conductor can be considered totally independent from the dissipation in the ground planes, for this specific geometry, when the envelope frequency increases. This allows the simplification of the model by considering only the center conductor in the thermal domain. With the equations in Section VI, a comparison between the contributions levels to the third-order intermodulation distortion, confirm the negligible contribution from the ground planes.

Fig. 7 also reveals that above a certain envelope frequency, a uniform temperature distribution in the center conductor, which is the main contribution to self-heating nonlinearities, can no longer be considered. The impact that the frequency-dependent temperature profile has on the distributed resistance is embedded in the frequency-dependent thermal impedance $Z_{\mathrm{th}}(\omega)$. Therefore, the model will provide an effective temperature rise at each frequency. In other words, the change produced in the distributed resistance by the existing temperature profile is the same as the change produced by a uniform effective temperature.

4) Thermal Impedance: A complete thermal-domain circuit implementation should be 3-D to consider heat flow along the $z$-axis, laterally and vertically through the substrate. The thermal domain implementation in Fig. 8 makes use of a series impedance $Z_{\Delta z, \mathrm{th}, S}(\omega)$, which models heat flow along the center conductor in the $z$ axis direction. It also includes a parallel impedance $Z_{\Delta z \text {,th,P }}(\omega)$ that models heat flow both laterally and vertically from the center conductor to the substrate.

The steady-state series and parallel thermal impedances are de-embedded by performing finite-element simulations with specifically implemented 3-D electrothermal finite-element software for different line lengths and for all different metals. The results are shown in Fig. 9, where we can observe that the parallel thermal impedance is roughly the same for all three metals. This indicates that the substrate has a dominating effect and is inversely proportional to length. On the other hand, the series impedance is different for all metals and is higher for poorer thermal conductors.

\section{Electro-Thermal Coupling}

Once the circuit implementations of both electromagnetic and thermal domains have been explained, the next step is to set the interaction between them. This interaction is a bidirectional process in the sense that the generated heat from the electromagnetic domain changes the temperature. This, in turn, changes the distributed resistance in the electromagnetic domain and, therefore, the generated heat.

The transmission line is implemented in $\Delta z$-long electrothermal cell segments. Heat generation by the Joule effect in 


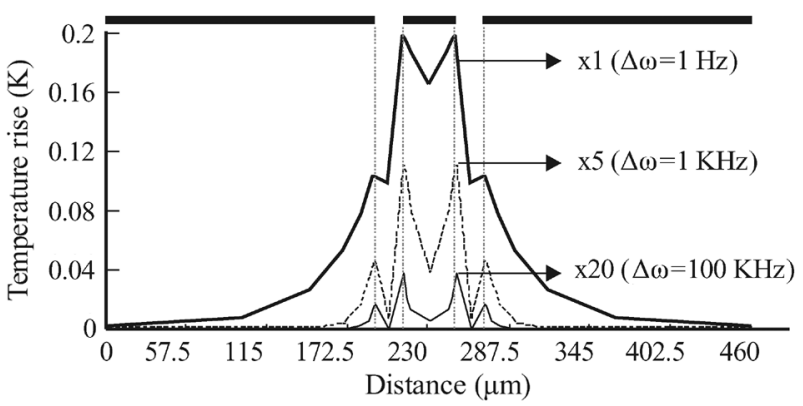

Fig. 7. Temperature distributions at different envelope frequencies. The plots have been scaled for better visualization purposes.

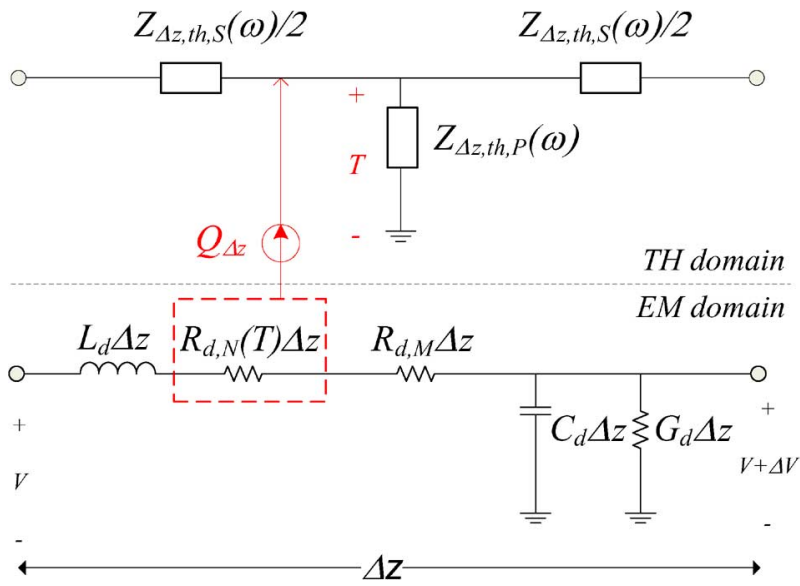

Fig. 8. Complete electrothermal circuit model of a $\Delta z$ segment of a transmission line.

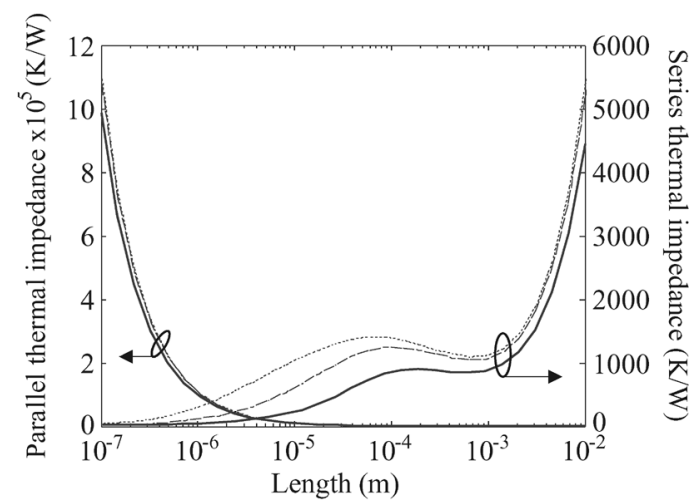

Fig. 9. Steady-state series and parallel thermal impedances as a function of length. The solid line represents $\mathrm{Au}$, whereas the dashed line represents $\mathrm{PdAu}$, and the dotted line represents Pt.

the center conductor at a certain position $z$ of the transmission line can be expressed as

$$
Q_{\Delta z}(z, t)=Q_{d}(z, t) \Delta z .
$$

Due to the analogy between heat and electric current in the heat equation, a current source is used to inject the generated heat $Q_{\Delta z}(z, t)$ in its correspondent thermal domain. Temperature rise, which is the analog of voltage in the thermal circuit domain, is then used to change the distributed resistance value $R_{d, N}(T)$.

The series thermal impedance has been checked to play a negligible role in the heat flow process by comparing the steady-state temperature rise along a transmission line with the model with $Z_{\Delta z, \mathrm{th}, S}(0)$ and with an open circuit instead of $Z_{\Delta z, \text { th }, S}(0)$ to finite-element simulations with specifically implemented 3-D electrothermal finite-element software, as shown in Section V.C-1. Therefore, a simplified model that makes use of only the parallel impedance will be used throughout this work.

The parallel impedance has a linear relation with length so that $Z_{\Delta z, \mathrm{th}, P}(\omega)=Z_{d, \mathrm{th}, P}(\omega) / \Delta z$, where $Z_{d, \mathrm{th}, P}(\omega)$ is the thermal impedance per unit length in the $z$ axis direction. The proposed circuit implementation of $Z_{\Delta z, \operatorname{th}, P}(\omega)$ is a resistance $R_{\Delta z \text {,th }}$ in parallel with a frequency-dependent capacitance $C_{\Delta z, \text { th }}(\omega)$ with $R_{\Delta z, \text { th }}=R_{d, \mathrm{th}} / \Delta z$ and $C_{\Delta z, \operatorname{th}}(\omega)=C_{d, \mathrm{th}}(\omega) \cdot \Delta z$. The term $R_{d, \mathrm{th}}$ in $\mathrm{K} \cdot \mathrm{m} / \mathrm{W}$ represents the steady-state distributed thermal resistance to heat flow. $C_{d, \mathrm{th}}(\omega)$ is the distributed volumetric heat capacity that embeds the frequency dispersive effects.

\section{CLOSED-FORM EXPRESSIONS}

Given the model presented in this work, closed-form expressions of the circuit implementation can be derived. In the present derivation, a matched transmission line is assumed despite extended expressions for mismatched transmission lines could be obtained by use of the procedure explained in [27].

\section{A. Heat Generation}

We consider a matched transmission line fed with two tones, $\omega_{1}$ and $\omega_{2}$, in close frequency. The current at each of the fundamental frequencies could be described as

$$
i_{n}(z, t)=\left|I_{n}\right| e^{-\alpha_{n} z} \cos \left(\omega_{n} t+\phi_{n}-\beta_{n} z\right)
$$

with $n=1,2$. In (9), $I_{n}=\left|I_{n}\right| e^{j \phi_{n}}$ and $\alpha_{n}, \beta_{n}$, and $\phi_{n}$ are the attenuation constant, phase constant, and phase, respectively. Therefore, the instantaneous dissipated power per unit length $Q_{d}(z, t)$, due to resistive losses in the center conductor, is

$$
Q_{d}(z, t)=\frac{\partial P_{d}(z, t)}{\partial z}=R_{d, N, T_{R}}\left(\sum_{n=1,2} i_{n}(z, t)\right)^{2}
$$

where $P_{d}(z, t)$ is the instantaneous propagating power, and as expected for a two-tone test, the quadratic nature of power dissipation leads to several spectral components

$$
\begin{aligned}
Q_{d}(z, t)=R_{d, N, T_{R}} & \\
\cdot \operatorname{Re}( & \frac{1}{2}\left|I_{1}\right|^{2} e^{-2 \alpha_{1} z}+\frac{1}{2}\left|I_{2}\right|^{2} e^{-2 \alpha_{2} z} \\
& +I_{1}^{*} I_{2} e^{j \Delta \omega t-\gamma_{v} z}+\frac{1}{2} I_{1}^{2} e^{j 2 \omega_{1} t-2 \gamma_{1} z} \\
& +\frac{1}{2} I_{2}^{2} e^{j 2 \omega_{2} t-2 \gamma_{2} z} \\
& \left.+I_{1} I_{2} e^{j\left(\omega_{1}+\omega_{2}\right) t-\left(\gamma_{1}+\gamma_{2}\right) z}\right)
\end{aligned}
$$

with $\gamma_{v}=\left(\alpha_{1}+\alpha_{2}\right)+j\left(-\beta_{1}+\beta_{2}\right)$, where $\gamma_{n}=\alpha_{n}+j \beta_{n}$ is the propagation constant of $I_{n}$.

As in the $3 \omega$-technique [15], we assume that heat flows mostly perpendicular to the substrate with a negligible heat flow along the $z$ axis of the line so only $Z_{d, \text { th }, P}(\omega)$ is considered. 
Equation (1), in which $Q_{d}(z, \omega)$ is the Fourier transform of $Q_{d}(z, t)$ at frequency $\omega$, states the physical relation between heat dissipation and temperature through the thermal impedance. Therefore, by use of the dissipated power at the envelope frequency $Q_{d}(z, \omega)=R_{d, N, T_{R}} I_{1}^{*} I_{2} e^{-\gamma_{v} z}$, the temperature distribution at the envelope frequency $\Delta \omega$ is

$$
T(z, \Delta \omega)=Z_{d, \mathrm{hh}, P}(\Delta \omega) R_{d, N, T_{R}} I_{1}^{*} I_{2} e^{-\gamma_{v} z} .
$$

We can provide the steady-state temperature along the line because of its impact on the linear performance of the device, where $Z_{d, \text { th }, P}(0)=R_{d, \text { th }}$

$$
T(z, 0)=R_{d, \mathrm{th}} R_{d, N, T_{R}}\left(\frac{1}{2}\left|I_{1}\right|^{2} e^{-2 \alpha_{1} z}+\frac{1}{2}\left|I_{2}\right|^{2} e^{-2 \alpha_{2} z}\right) .
$$

\section{B. Temperature-Induced Intermodulation Distortion}

As previously stated, a temperature-independent dielectric is considered so that only the $\Delta R_{d, N}$ term that appears in (5) is responsible for the nonlinear behavior so long as it quantifies the temperature dependence of the distributed resistance. Therefore, for a matched transmission line with negligible heat propagation along $z$, the nonlinear voltage per unit length can be obtained by introducing (5) in (3) and separating the resulting equation in a linear term and a nonlinear term, where the nonlinear term is

$$
\frac{\partial v_{n l}(z, t)}{\partial z}=\Delta R_{d, N} T(z, t) i(z, t)
$$

Considering the case of a two-tone test in which the line is driven by two different tones (9) at frequencies $\omega_{1}$ and $\omega_{2}$, the frequency component $\omega_{12}=2 \omega_{1}-\omega_{2}$ of (14) would be

$$
\frac{\partial V_{n l, \omega_{12}}(z)}{\partial z}=\frac{1}{2} \Delta R_{d, N} T^{*}(z, \Delta \omega) I_{1} e^{-\gamma_{1} z}
$$

where $T(z, \Delta \omega)$ is the temperature oscillations at the envelope frequency $\Delta \omega$. By substituting (12) into (15), we obtain

$$
\frac{\partial V_{n l, \omega_{12}}}{\partial z}=\frac{1}{2} \Delta R_{d, N} I_{1}^{2} I_{2}^{*} Z_{d, \mathrm{th}, P}^{*}(\Delta \omega) R_{d, N, T_{R}} e^{-\gamma_{c} z}
$$

with $\gamma_{c}=\left(2 \alpha_{1}+\alpha_{2}\right)+j\left(2 \beta_{1}-\beta_{2}\right)$. If we combine the telegrapher equations

$$
\begin{array}{r}
\frac{\partial^{2} i}{\partial z^{2}}=L_{d} C_{d} \frac{\partial^{2} i}{\partial t^{2}}+\left(R_{d} C_{d}+L_{d} G_{d}\right) \frac{\partial i}{\partial t}+R_{d} G_{d} i \\
+C_{d} \frac{\partial^{2} v_{n l}}{\partial z \partial t}+G_{d} \frac{\partial v_{n l}}{\partial z}
\end{array}
$$

which can be rewritten as

$$
\frac{\partial^{2} I_{i}(z)}{\partial z^{2}}=\gamma_{i}^{2} I_{i}(z)+\frac{\gamma_{i}}{Z_{i}} \frac{\partial V_{n l, i}}{\partial z}
$$

where $i=1,2$ refers to frequencies $\omega_{1}$ and $\omega_{2}$ with

$$
\gamma_{i}=\sqrt{\left(R_{d, i}+j \omega_{i} L_{d}\right)\left(G_{d}+j \omega_{i} C_{d}\right)}
$$

and

$$
Z_{i}=\sqrt{\frac{R_{d, i}+j \omega_{i} L_{d}}{G_{i}+j \omega_{i} C_{d}}} .
$$

We now substitute (16) in (18) to get

$$
\begin{aligned}
& \frac{\partial^{2} I_{12}(z)}{\partial z^{2}}=\gamma_{12}^{2} I_{12}(z)+\frac{\gamma_{12}}{Z_{12}} \frac{1}{2} \Delta R_{d, N} I_{1}^{2} I_{2}^{*} Z_{d, \text { th }, P}^{*} \\
& \times(\Delta \omega) R_{d, N, T_{R}} e^{-\gamma_{c} z}
\end{aligned}
$$

where the subscript 12 refers to frequency $\omega_{12}=2 \omega_{1}-\omega_{2}$. Equation (21) can be solved for the nonlinear current along the line

$$
\begin{aligned}
I_{12}(z)=\frac{1}{2 Z_{12}} \Delta R_{d, N} I_{1}^{2} I_{2}^{*} & R_{d, N, T_{R}} Z_{d, \mathrm{th}, P}^{*}(\Delta \omega) \\
& \times \frac{\gamma_{12}\left(e^{-\gamma^{\prime} z}-1\right)}{\gamma^{\prime}\left(\gamma^{\prime}+2 \gamma_{12}\right)} e^{-\gamma_{12} z}
\end{aligned}
$$

with

$$
\gamma^{\prime}=\gamma_{c}-\gamma_{12}=\left(2 \alpha_{1}+\alpha_{2}-\alpha_{12}\right)+j\left(2 \beta_{1}-\beta_{2}-\beta_{12}\right) .
$$

Equation (22) can be approximated to

$$
\begin{array}{r}
I_{12}(z)=\frac{1}{8 Z_{12}} \Delta R_{d, N} I_{1}^{2} I_{2}^{*} R_{d, N, T_{R}} Z_{d, \mathrm{th}, P}^{*}(\Delta \omega) \\
\times \frac{\left(e^{-2 \alpha_{1} z}-1\right)}{\alpha_{1}} e^{-\gamma_{12} z} .
\end{array}
$$

Thus, if we assume $\left|I_{1}\right|=\left|I_{2}\right|$, the power delivered to a matched load is

$$
P_{L, 12}=k_{12}\left|\Delta R_{d, N} Z_{d, \mathrm{th}, P}(\Delta \omega) R_{d, N, T_{R}}\right|^{2}\left|I_{1}\right|^{6}
$$

with

$$
k_{12}=\frac{\left(e^{-2 \alpha_{0} l}-1\right)^{2} \operatorname{Re}\left(Z_{12}\right)}{128\left|Z_{12}\right|^{2} \alpha_{0}^{2}} e^{-2 \alpha_{12} l} .
$$

\section{Validation of Expressions With Simulations}

The above-presented expressions are validated by making use of circuit simulations with the electrothermal model implemented with 100 cells, such as the one shown in Fig. 8. Both the equations and electromagnetic domain of the circuit model use the distributed parameters of the gold line previously used in the thermal analysis.

Though understanding the intermodulation process is the final goal of this work, an important preliminary step consists in checking the temperature rise. Simulations with the model have been performed by using two tones, at $20 \mathrm{dBm}$ each, and at variable frequency spacing between them. The upper tone is fixed at $6 \mathrm{GHz}$. The same frequency-dependent thermal impedance $Z_{d, \mathrm{th}, P}(\omega)$ is used on both equations and simulation.

Equation (13), which gives the steady-state temperature distribution along the $z$ axis, has been checked with a matched transmission line, resulting in perfect agreement. To better il- 


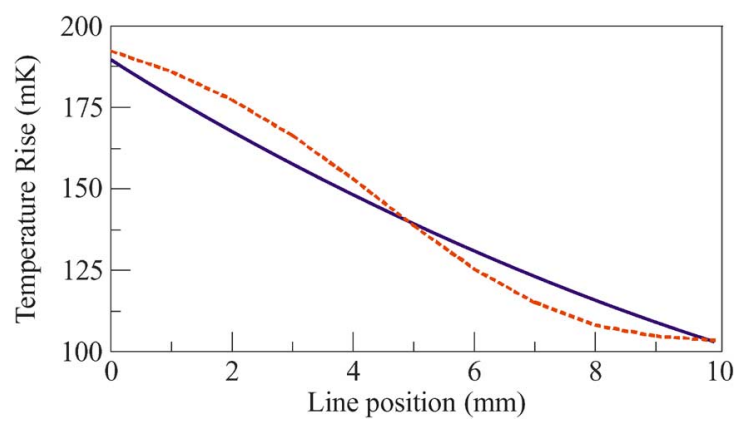

Fig. 10. Steady-state temperature rise along the $9.93-\mathrm{mm}$ gold line. Solid line is using (13) and dashed line represents the results from simulation.

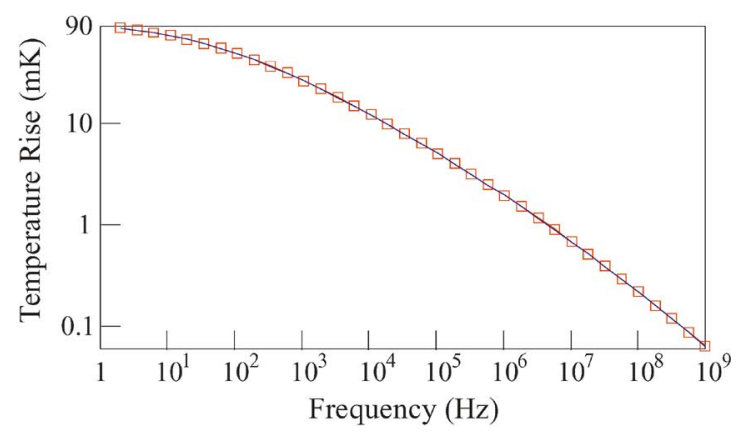

Fig. 11. Temperature rise at $z=9.93 \mathrm{~mm}$ of a $9.93-\mathrm{mm}$ gold line for a wide range of envelope frequencies. Squares and solid line are simulation and equation, respectively.

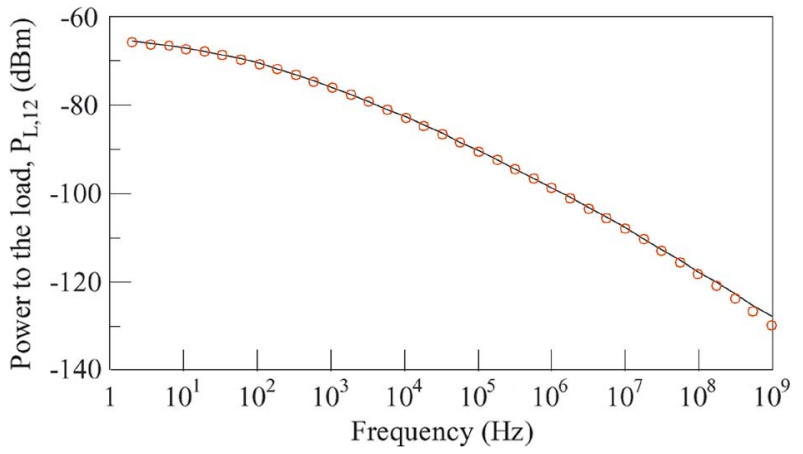

Fig. 12. Power delivered to a matched load at the end of the $9.93-\mathrm{mm}$ gold line. Circles and solid line are simulation and equation respectively.

lustrate this with a real case, Fig. 10 shows the results for (13) and the circuit model implementation of a $9.93-\mathrm{mm}$ gold line, and $\Delta \omega=100 \mathrm{~Hz}$, where simulations give a small ripple due to the imaginary part of $Z_{0}=50.35-j 2 \Omega$. For the real 9.93-mm gold line circuit implementation, distributed parameters extracted from measurements are used to construct the transmission line as a cascade of cells.

Additionally, temperature rise oscillations are validated, by use of (12) and simulations, at different envelope frequencies at $z=9.93 \mathrm{~mm}$ (Fig. 11).

Once the equations of the temperature rise have been validated, the nonlinear signal, generated as a consequence of temperature oscillations, can be evaluated. Fig. 12 shows the result of (25) and simulations of the intermodulation power delivered to a matched load at $z=9.93 \mathrm{~mm}$ for a wide range of envelope frequencies.

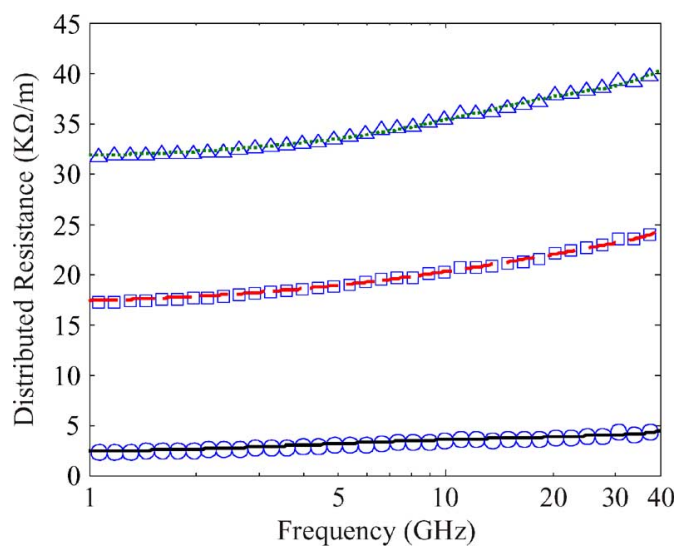

Fig. 13. Extracted distributed resistance (symbols) and polynomial fit (lines) for the different coplanar waveguides. Circles and solid line represent gold, squares and dashed line represent platinum, and triangles and dotted line represent palladium-gold.

\section{Measurements AND Results}

A test wafer has been constructed to validate the model with measurements. Metals with different temperature coefficients of resistivity have been selected to construct coplanar waveguides of different lengths. To check the model, measurements of the resistivity at different temperatures have been performed. Thirdorder intermodulation distortion measurements for a wide range of envelope frequencies have also been used.

\section{A. Test Wafer}

Coplanar waveguides, with the cross-section geometry shown in Fig. 3, have been constructed following standard fabrication techniques on a sapphire substrate, chosen for its low dielectric losses at microwave frequencies. Different metals have been deposited, including gold $(\mathrm{Au})$, platinum $(\mathrm{Pt})$, and palladium-gold (PdAu) with a 55\% gold content. The reason for choosing these metals is that they offer different combinations of resistivity values and resistivity change with temperature, which translates into different levels of intermodulation distortion. Three transmission lines, $\mathrm{A}, \mathrm{B}$, and $\mathrm{C}$ for each type of metal, have been measured. Their lengths are $l_{A}=1 \mathrm{~mm}, l_{B}=4.2 \mathrm{~mm}$, and $l_{C}=9.93 \mathrm{~mm}$, to demonstrate the distributed effects.

\section{B. Linear Measurements and Model}

The first step in constructing the linear part of the circuit model is to obtain the distributed parameters of the fabricated transmission lines. The procedure used in this work consists in performing a multiline thru-reflect-line (TRL) calibration to obtain the propagation constant. It is then used, along with an impedance comparison method, to obtain the distributed parameters $R_{d, T_{R}}, L_{d}, C_{d}$, and $G_{d}$. Details on this procedure can be found in [28].

The linear measurements have been performed at a low power, $-5 \mathrm{dBm}$, to ensure the linear regime of the devices. Results for the extracted distributed resistance and inductance are shown in Figs. 13 and 14, respectively.

The extracted values of the distributed conductance $G_{d}$ are below the sensitivity of the measurements; its impact is, there- 


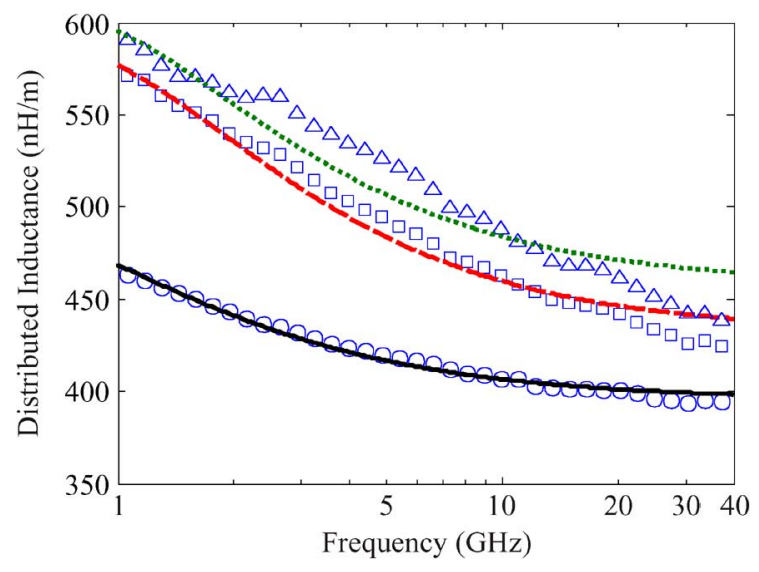

Fig. 14. Extracted distributed inductance (symbols) and polynomial fit (lines) for the different coplanar waveguides. Circles and solid line represent gold, squares and dashed line represent platinum, and triangles and dotted line represent palladium-gold.

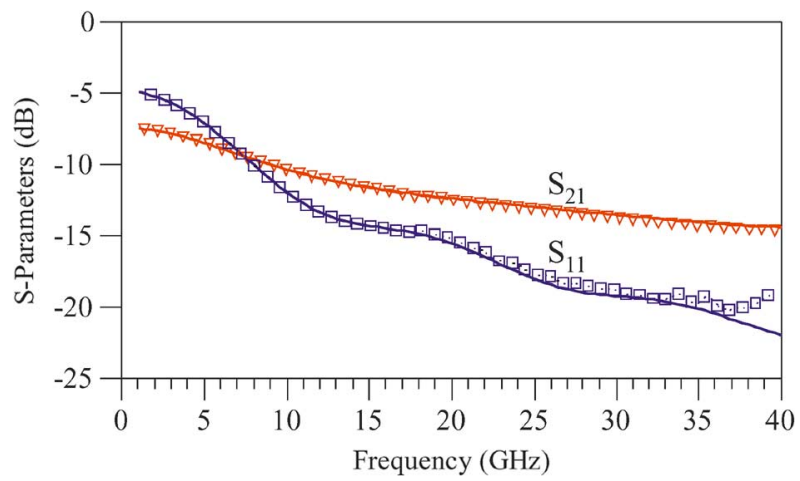

Fig. 15. Measured and simulated $S$-parameters for line B, made of palladiumgold. The line length is $4.2 \mathrm{~mm}$. Squares and triangles represent measurements. Solid lines represent simulation.

fore, considered negligible. On the other hand, the extracted distributed capacitance, which is constant over frequency due to the nondispersive nature of sapphire, has a value of $C_{d}=162 \mathrm{pF} / \mathrm{m}$.

The presented extracted distributed resistance and inductance are fitted to polynomials so that they can be easily used in the circuit model implementation. Next, the calibrated $S$-parameters from measurements are compared to the simulated $S$-parameters of the circuit implementation for each line to check that the extraction procedure is correct.

For example, Fig. 15 illustrates both measured and simulated $S$-parameters for PdAu line B. Characteristic impedances obtained at $6 \mathrm{GHz}$ are $Z_{0, \mathrm{Au}}=50.35-j 2 \Omega, Z_{0, \mathrm{Pt}}=60.16-$ $j 25.64 \Omega$, and $Z_{0, \mathrm{PdAu}}=69.53-j 40.29 \Omega$.

\section{Nonlinear Measurements and Model}

Once the linear measurements and modeling are complete, the thermal impedance that correctly predicts the temperature along the line is obtained. An accurate temperature simulation is crucial to properly simulate its effect on the metal resistivity and also to predict the third-order intermodulation generation. In the model presented, the thermal resistance $R_{d \text {,th }}$ and the thermal capacitance per unit length $C_{d, \mathrm{th}}(\omega)$ represent the resistance to heat flow at steady state and its frequency dependence, respectively. These can, therefore, be solved separately. As stated
TABLE II

Electric AND Thermal PARAmeters of the Center Conductor AT $z=0$ USING TWO TONES AT $20 \mathrm{dBm}$ EACH

\begin{tabular}{lccc}
\hline \hline & $\mathrm{Au}$ & $\mathrm{Pt}$ & $\mathrm{PdAu}$ \\
\hline $\mathrm{I}_{\text {Total }}(\mathrm{A})$ & $6.4 \cdot 10^{-2}$ & $5.6 \cdot 10^{-2}$ & $5.1 \cdot 10^{-2}$ \\
\hline $\mathrm{Q}_{\mathrm{d}}\left(\mathrm{W} \cdot \mathrm{m}^{-1}\right)$ & 9.26 & 50.3 & 80.5 \\
\hline $\mathrm{T}_{\mathrm{Av}}(\mathrm{K})$ & 0.29 & 1.6 & 2.65 \\
\hline $\mathrm{R}_{\mathrm{d}, \mathrm{th}}(\mathrm{K} \cdot \mathrm{m} / \mathrm{W})$ & $3.13 \cdot 10^{-2}$ & $3.18 \cdot 10^{-2}$ & $3.29 \cdot 10^{-2}$ \\
\hline \hline
\end{tabular}

in (2), the third-order intermodulation measurements unveil the frequency dependence of the thermal impedance, which is used in this work to obtain $C_{d, \mathrm{th}}(\omega)$. On the other hand, finite-element thermal simulations are used to obtain $R_{d, \text { th }}$.

1) Finite-Element Thermal Simulations: The thermal resistance $R_{d, \text { th }}$ sets the steady-state temperature, given a certain heat dissipation in the center conductor. It depends not only on the material properties of the metal and substrate, but also the geometry of the strip. Finite-element simulations can be used to obtain the thermal resistance $R_{d, \text { th }}$ with (1) as the division of temperature over heat.

We look at the heat density through the line, shown in Fig. 4, and perform the surface integral at the center conductor. A steady-state thermal simulation is done with [23] to obtain the temperature profile on the cross section of the line. Table II summarizes the current $I_{\text {Total }}$ and heat flux $Q_{d}$ at $z=0$ for all types of metal transmission lines, the average temperature rise on the center conductor $T_{\mathrm{AV}}$, and the thermal resistance $R_{d, \mathrm{th}}$ obtained.

As can be seen from Table II, the change in the thermal resistance $R_{d, \text { th }}$ is around $5 \%$ for the three metals. This implies that the upper metal layer plays a negligible role in how the heat flows to the substrate. Approximate values for $R_{d, \text { th }}$ might be obtained with closed-form expressions such as those found in [13] and [14] to get $R_{d, \text { th }}=0.026 \mathrm{~K} \cdot \mathrm{m} / \mathrm{W}$ and $R_{d, \text { th }}=$ $0.028 \mathrm{~K} \cdot \mathrm{m} / \mathrm{W}$, respectively, neglecting the titanium adhesion layer.

The circuit model can also be used to simulate the temperature distribution along the line. To check this, we performed 3-D steady-state thermal finite-element simulations with specifically implemented 3-D electrothermal finite-elelent software. This is done by use of the current distribution previously obtained with the circuit model, as shown in Fig. 16.

Results in Fig. 16 show the contribution to temperature rise due to dissipation in the center conductor for the circuit model and finite-element simulations. There is total agreement between them. Moreover, Fig. 16 also shows the total temperature rise in the center conductor as a consequence of dissipation in the center conductor and the ground planes obtained with finite-element simulations. The simulations for the circuit model have been performed with and without the series thermal impedance $Z_{\Delta z, \mathrm{th}, S}(\omega)$, which totally overlap.

These results confirm that, for the given geometry, the heat propagation along the $z$ axis is negligible and the circuit model can be used to estimate the total temperature rise in the center conductor.

2) Third-Order Intermodulation Distortion: Forward thirdorder intermodulation distortion measurements, of $2 \omega_{1}-\omega_{2}$ and $2 \omega_{2}-\omega_{1}$, have been performed on all lines by means of the 


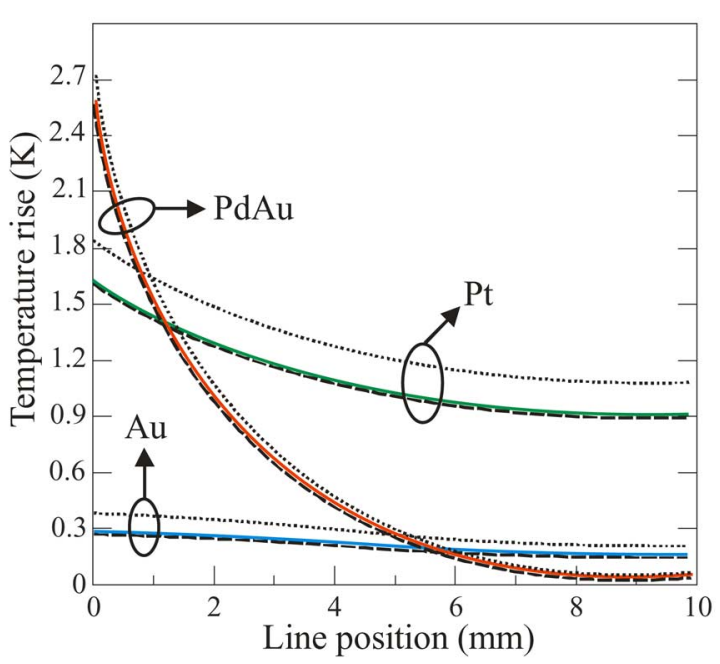

Fig. 16. Steady-state temperature rise profiles along the $\mathrm{C}$ lines for the different metals. Solid and dashed lines represent the contribution to temperature rise in the center conductor, as a consequence of its dissipation, from using the circuit model and finite elements, respectively. The dotted lines represent the total temperature rise in the center conductor, as a consequence of dissipation in both the center conductor and the ground planes, obtained with finite-element simulations. Simulations using the circuit model, with $Z_{\Delta z, \text { th }, S}(\omega)$ and with an open circuit instead of $Z_{\Delta z, \mathrm{th}, S}(\omega)$, overlap.

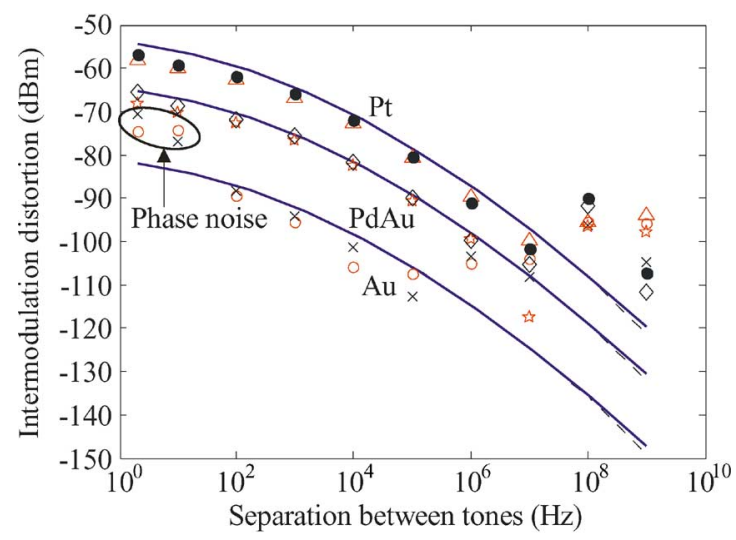

Fig. 17. Measured and simulated third-order intermodulation in A line, $l_{\mathrm{A}}=$ $1 \mathrm{~mm} .2 f_{1}-f_{2}$ (dashed line) and $2 f_{2}-f_{1}$ (solid line) overlap for all the simulations. Unfilled circles and crosses represent $2 f_{1}-f_{2}$ and $2 f_{2}-f_{1}$ for Au. Triangles and filled circles represent $2 f_{1}-f_{2}$ and $2 f_{2}-f_{1}$ for Pt. Stars and diamonds represent $2 f_{1}-f_{2}$ and $2 f_{2}-f_{1}$ for PdAu.

two-tone test [10]. A special measurement setup [28] consisting of a configuration that cancels the fundamental signals $\omega_{1}$ and $\omega_{2}$ after the device-under-test is used to achieve a high-dynamic range at the spectrum analyzer. For such measurements, the input power has been fixed at $20 \mathrm{dBm}$, while the tones spacing has been taken from $2 \mathrm{~Hz}$ up to $1 \mathrm{GHz}$. Results can be seen in Figs. 17-19.

High separation between tones measurements are limited by the baseline intermodulation level of the measurement setup. On the other hand, small separations between tones measurements can be limited by the phase noise of the sources. The shortest $\mathrm{Au}$ line represents the worst scenario for this type of measurements because it shows a low intermodulation level. System nonlinearities easily dominate at intermediate and high separation between tones $\left(\Delta f>10^{5} \mathrm{~Hz}\right)$ and phase noise

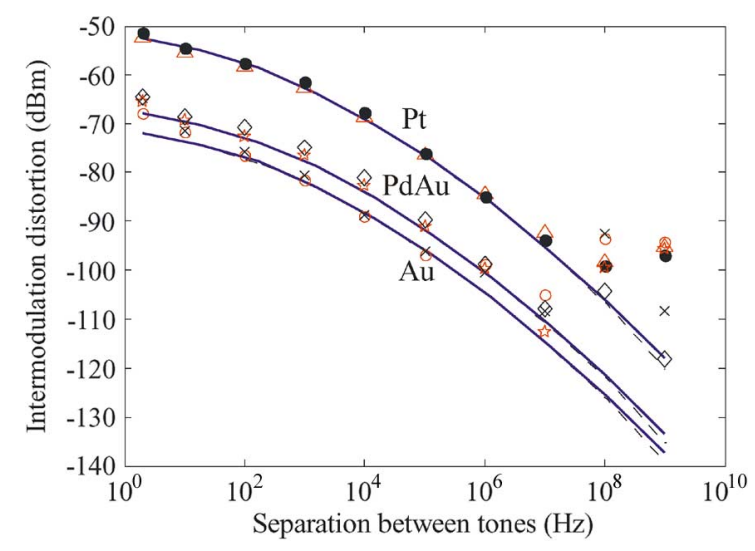

Fig. 18. Measured and simulated third-order intermodulation in $\mathrm{B}$ line, $l_{\mathrm{B}}=$ $4.2 \mathrm{~mm} .2 f_{1}-f_{2}$ (dashed line) and $2 f_{2}-f_{1}$ (solid line) overlap for all the simulations. Unfilled circles and crosses represent $2 f_{1}-f_{2}$ and $2 f_{2}-f_{1}$ for $\mathrm{Au}$. Triangles and filled circles represent $2 f_{1}-f_{2}$ and $2 f_{2}-f_{1}$ for Pt. Stars and diamonds represent $2 f_{1}-f_{2}$ and $2 f_{2}-f_{1}$ for PdAu.

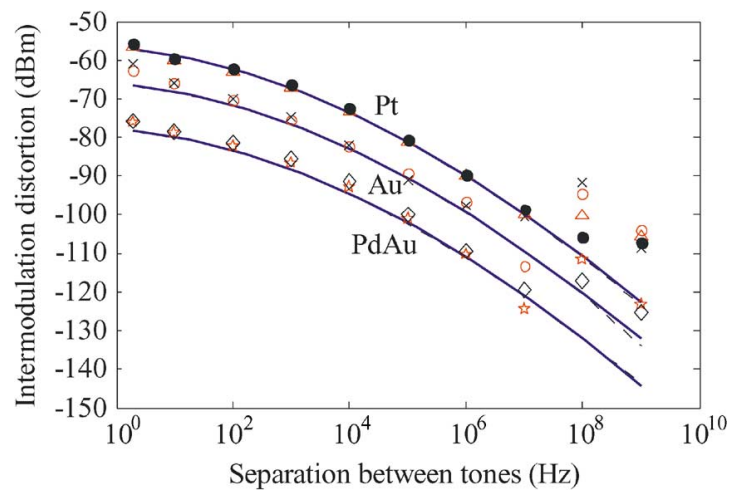

Fig. 19. Measured and simulated third-order intermodulation in $\mathrm{C}$ line, $l_{\mathrm{C}}=$ $9.93 \mathrm{~mm} .2 f_{1}-f_{2}$ (dashed line) and $2 f_{2}-f_{1}$ (solid line) overlap for all the simulations. Unfilled circles and crosses represent $2 f_{1}-f_{2}$ and $2 f_{2}-f_{1}$ for Au. Triangles and filled circles represent $2 f_{1}-f_{2}$ and $2 f_{2}-f_{1}$ for Pt. Stars and diamonds represent $2 f_{1}-f_{2}$ and $2 f_{2}-f_{1}$ for PdAu.

dominates for small separation between tones $\left(\Delta f<10^{2} \mathrm{~Hz}\right)$. The results in Figs. 17-19 unveil the low-pass filter behavior of the thermal impedance, showing similar results as those found in [12], which can be used to extract the frequency-dependent thermal capacitance $C_{d, \operatorname{th}}(\omega)$. Measurements of any of the metals could be used to extract $C_{d, \text { th }}(\omega)$, as long as the thermal impedance is dominated by the substrate. The platinum $\mathrm{B}$ line is preferable, though, because it shows the highest dynamic range above the baseline intermodulation level of the measurement setup, which is around $-100 \mathrm{dBm}$. The extracted phenomenological $C_{d, \mathrm{th}}(\omega)$, for the procedure explained in Appendix II, can be seen in Fig. 20, where its frequency-dependence embeds the 3-D dissipation effect.

Once the thermal model is completely implemented, the last step consists of determining the nonlinear variable $R_{d, N}(T)$. This sets how the distributed resistance of the center conductor changes with temperature. To do that, we use the relation between $R_{d, N}(T)$ and temperature, presented in Appendix I, so that the temperature coefficient of resistivity of the metal is used. Since the material properties are process dependent, we performed resistance measurements at dc of the fabricated coplanar waveguides at several temperatures. 


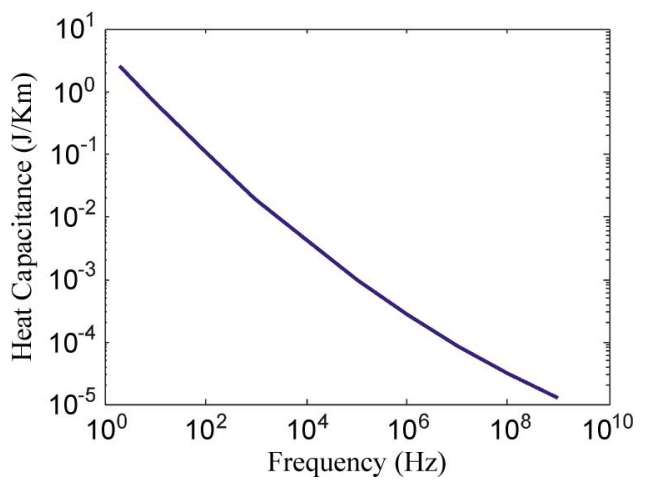

Fig. 20. Extracted $C_{d, \text { th }}(\omega)$ from measurements on the platinum B line.

The measured values for the temperature coefficients of resistivity are $\delta_{\mathrm{Au}}=0.0039987 \mathrm{~K}^{-1}, \delta_{\mathrm{Pt}}=0.002824 \mathrm{~K}^{-1}$, and $\delta_{\mathrm{PdAu}}=0.000372 \mathrm{~K}^{-1}$. The resistivity values at room temperature are shown in Table I.

The complete electrothermal model can now be constructed, making use of the measured $\delta$ values and the extracted thermal resistance and capacitance. Simulations of the third-order intermodulation distortion with the model are presented, along with the measurements in Figs. 17-19. These show good agreement and the predicted dependence on the line length.

\section{DISCUSSION}

The good agreement obtained between measurements and modeling indicates that scaling with the line length is correctly predicted. From the results above, the high difference between nonlinear levels on lines made of different metals can also be observed. The results show that there is not a simple relation between length and intermodulation level, and this is because the distributed nonlinearity increases with length, but also gets attenuated by it. Therefore, depending on the length, a high attenuation transmission line can show more or less nonlinearity than a lower loss transmission line. In addition, the fact that the platinum and the palladium-gold lines are highly mismatched translates into a variable relation between length and intermodulation level [27].

However, several considerations to minimize the intermodulation can be made from (25), in particular that referred to the correct choice of materials. In this sense, metals and dielectrics with low losses and weakly temperature-dependent properties are preferable. Additionally, high thermal conductivity dielectrics provide the right path for heat, minimizing temperature rise.

To check that the ground planes have a negligible contribution to the intermodulation distortion generation, we use (25). We consider that each ground metal strip has the same thermal impedance of the center conductor, which is a reasonable approximation. With this assumption, we get a much lower contribution coming from the ground planes of $\Delta 3 \mathrm{IMD}_{\mathrm{Au}}=10 \log \left(\left|P_{L, 12, M} / P_{L, 12, N}\right|_{\mathrm{Au}}\right)=-27.1 \mathrm{~dB}$ when compared with that coming from the center conductor in the gold lines. For the platinum and palladium-gold lines, we get $\Delta 3 \mathrm{IMD}_{\mathrm{Pt}}=-42 \mathrm{~dB}$ and $\Delta 3 \mathrm{IMD}_{\mathrm{PdAu}}=-48.4 \mathrm{~dB}$. These results confirm the validity of the simplified model.
From the closed-form expressions obtained, we observe that the third-order intermodulation distortion generation process might also be described, for a specific separation between tones, by a phenomenological model of a quadratic current-dependent distributed resistance of the form

$$
R_{d}(I)=R_{d, 0}+R_{d, 2} I^{2} .
$$

Several authors have suggested the use of (27) to explain the relation between losses and intermodulation [16]. However, this phenomenological model is incorrect, and predicts a nonexistent third harmonic.

\section{CONCLUSIONS}

We have presented the mechanism by which third-order intermodulation distortion is generated in transmission lines and its circuit model. Additionally, closed-form expressions have been obtained and validated along with the model. The model has been properly checked with measurements of lines of different lengths and made composed of different metals.

This work also reveals the negligible impact of the ground planes when compared to that of the center conductor and in terms of nonlinear behavior due to self-heating. Additionally, the envelope frequency-dependent intermodulation cannot be described by a constant slope of a certain decibel/decade.

The advantage of having such a distributed circuit model for a nonlinear transmission line is that it can be used for prediction purposes of any device in which transmission lines are used such as filters or directional couplers. Moreover, its usefulness is not restricted to a specific kind of input signal and can predict the nonlinear effects on complex modulated signals like global system for mobile communication (GSM) or code division multiple access (CDMA) for example.

\section{APPENDIX I}

\section{Distributed Resistance TEMPERATURE DEPENDENCE}

The distributed resistance at a specific frequency can be described as a function of the resistivity increment as follows:

$$
R_{d}=R_{d, T_{R}}(1+\gamma \Delta \rho(T))
$$

where $R_{d, T_{R}}$ is the distributed resistance at ambient temperature. The resistivity increment due to temperature rise $\Delta \rho(T)$ can be identified in the following relation:

$$
\rho(T)=\rho_{T_{R}}+\Delta \rho(T)=\rho_{T_{R}}(1+\delta \cdot T) .
$$

$\gamma$ is the local derivative, at the room temperature resistivity value, of the function that relates the distributed resistance with the metal resistivity. Therefore, $\gamma$ is a factor that relates the increment in the distributed resistance, as a consequence of an increment in the resistivity, for a specific geometry and metal

$$
\gamma=\left.\frac{1}{R_{d, T_{R}}} \frac{\partial R_{d}(\rho)}{\partial \rho}\right|_{\rho_{T_{R}}} .
$$

By using the Weeks method [29], we obtain the $\gamma$ factor for the center conductor, for the given geometry at $6 \mathrm{GHz}$ to be 
$\gamma_{\mathrm{Au}}=2.98 \cdot 10^{7} \Omega^{-1} \cdot \mathrm{m}^{-1}, \gamma_{\mathrm{Pt}}=4.12 \cdot 10^{6} \Omega^{-1} \cdot \mathrm{m}^{-1}$, and $\gamma_{\mathrm{PdAu}}=2.24 \cdot 10^{6} \Omega^{-1} \cdot \mathrm{m}^{-1}$.

\section{APPENDIX II}

\section{THERMal CAPACITANCE EXTRACTION PROCEDURE}

The procedure starts by converting the intermodulation measurements to a linear scale to get the unscaled magnitude of the thermal impedance $\left|Z_{u, \text { th }, P}(\omega)\right| .\left|Z_{u, \text { th }, P}(\omega)\right|$ is then fitted to a polynomial and scaled to a value previously obtained using the circuit model to get $\left|Z_{d, \text { th,P }}(\omega)\right|$. A frequency-dependent thermal capacitance of the form

$$
C_{d, \mathrm{th}}(\omega)=10^{C_{\mathrm{th}, 0}+C_{\mathrm{th}, 1} \log (\omega)+C_{\mathrm{th}, 2} \log (\omega)^{2}}
$$

is used so that its coefficients can be obtained from the relation that follows from a simple circuit analysis of Fig. 8:

$$
C_{d, \mathrm{th}}(\omega)=\frac{1}{\omega} \sqrt{\frac{1}{\delta z^{2}\left|Z_{d, \mathrm{th}, P}(\omega)\right|^{2}}-\frac{1}{R_{d, \mathrm{th}}^{2}}} .
$$

The extracted phenomenological $C_{d, \text { th }}(\omega)$ has coefficients $C_{\mathrm{th}, 0}=5.0868, C_{\mathrm{th}, 1}=-0.14$, and $C_{\mathrm{th}, 2}=0.0007$.

\section{ACKNOWLEDGMENT}

The authors thank Dr. Y. Wang and Dr. T. M. Wallis, both with the National Institute of Standards and Technology (NIST), Boulder, $\mathrm{CO}$, for their help with this work.

Work partially supported by the U.S. Government not subject to U.S. copyright.

\section{REFERENCES}

[1] C. Collado, J. Mateu, and J. M. O'Callaghan, "Analysis and simulation of the effects of distributed nonlinearities in microwave superconducting devices," IEEE Trans. Appl. Superconduct., vol. 15, no. 1, pp. 26-39, Mar. 2005.

[2] J. Mateu, J. C. Booth, S. A. Schima, C. Collado, D. Seron, and J. M. O'Callaghan, "Measurements and analysis of microwave nonlinearities in ferroelectric thin film transmission lines," in IEEE MTT-S Int. Microw. Symp. Dig., Jun. 11-16, 2006, pp. 1622-1625.

[3] A. P. Shitvov, D. E. Zelenchuk, A. G. Schuchinsky, and V. F. Fusco, "Passive intermodulation in printed lines: Effects of trace dimensions and substrate," IET Microw., Antennas, Propag., vol. 3, no. 2, pp. 260-268, Mar. 2009.

[4] D. E. Zelenchuk, A. P. Shitvov, and A. G. Schuchinsky, "Effect of laminate properties on passive intermodulation generation," in LAPC Antennas Propag. Conf., Apr. 2-3, 2007, pp. 169-172.

[5] P. L. Lui, "Passive intermodulation interference in communication systems," Electron. Commun. Eng. J., vol. 2, no. 3, pp. 109-118, Jun. 1990.

[6] P. N. Radha, "Predicting junction temperature and MTTF for MMIC devices," RF Design, vol. 28, no. 8, pp. 68-70, 2005.

[7] R. Hopper and C. Oxley, "Thermal measurement a requirement for monolithic microwave integrated circuit design," in Proc. ARMMS, Apr. 2008, pp. 385-390.

[8] V. Camarchia, F. Cappelluti, M. Pirola, S. D. Guerrieri, and G. Ghione, "Self-consistent electrothermal modeling of class A, AB, and B power GaN HEMTs under modulated RF excitation," IEEE Trans. Microw. Theory Tech., vol. 55, no. 9, pp. 1824-1831, Sep. 2007.

[9] A. E. Parker and J. G. Rathmell, "Self-heating process in microwave transistors," presented at the Appl. Radio Sci. Workshop, Hobart, TAS, Australia, Feb. 18-20, 2004.
[10] J. H. K. Vuolevi, T. Rahkonen, and J. P. A. Manninen, "Measurement technique for characterizing memory effects in RF power amplifiers," IEEE Trans. Microw. Theory Tech., vol. 49, no. 8, pp. 1383-1389, Aug. 2001.

[11] R. S. Timsit, "High speed electronic connectors: A review of electrical contact properties," IEICE Trans. Electron., vol. ESS-C, no. 8, pp. 1532-1544, 2005.

[12] J. R. Wilkerson, K. G. Gard, A. G. Schuchinsky, and M. B. Steer, "Electro-thermal theory of intermodulation distortion in lossy microwave components," IEEE Trans. Microw. Theory Tech., vol. 56, no. 12, pp. 2717-2725, Dec. 2008.

[13] R. Wilcoxon, "The effects of geometry and dielectric material on stripline and microstrip internal temperatures," IEEE Trans. Compon. Packag. Technol., vol. 28, no. 4, pp. 674-679, Dec. 2005.

[14] J. Adam, "New correlations between electrical current and temperature rise in PCB traces," in 20th Annu. IEEE Semiconduct. Thermal Meas. Manag. Symp., Mar. 9-11, 2004, pp. 292-299.

[15] D. G. Cahill, "Thermal conductivity measurement from 30-750 K: The $3 \omega$ method," Rev. Sci. Instrum., vol. 61, pp. 802-808, 1990.

[16] D. E. Zelenchuk, A. P. Shitvov, A. G. Schuchinsky, and V. F. Fusco, "Passive intermodulation in finite lengths of printed microstrip lines," IEEE Trans. Microw. Theory Tech., vol. 56, no. 11, pp. 2426-2434, Nov. 2008.

[17] J. Z. Wilcox and P. Molmud, "Thermal heating contribution to intermodulation fields in coaxial waveguides," IEEE Trans. Commun., vol. COM-24, no. 2, pp. 238-243, 1976.

[18] G. H. Strauss, "Intrinsic sources of IM generation," Naval Res. Lab., CITY, STATE, Memo. Rep. 4233, 1980, ch. 5.

[19] E. Rocas, C. Collado, N. D. Orloff, and J. C. Booth, "Third order intermodulation distortion due to self-heating in gold coplanar waveguides," in IEEE MTT-S Int. Microw. Symp. Dig., May 2010, pp. 425-428.

[20] F. P. Incropera, D. P. DeWitt, T. L. Berqman, and A. S. Lavine, Fundamentals of Heat and Mass Transfer. New York: Wiley, 2002.

[21] C. Collado, J. Mateu, O. Menendez, and J. M. O'Callaghan, "Nonlinear distortion in a 8-pole quasi-elliptic bandpass HTS filter for CDMA system," IEEE Trans. Appl. Superconduct., vol. 15, no. 2, pp. 992-995, Jun. 2005.

[22] D. Pozar, Microwave Engineering. New York: Wiley, 1998.

[23] QuickField. Tera Software, Svendborg, Denmark, 2010.

[24] "Material property data," MatWeb, Blacksburg, VA, 2010. [Online]. Available: http://www.matweb.com

[25] "Characteristics of single crystal sapphire," KYOCERA, Kyoto, Japan, 2010. [Online]. Available: http://global.kyocera.com/prdct/fc/product/ pdf/s_c_sapphire.pdf

[26] C. Y. Ho, "Thermal conductivity of ten selected binary alloy systems," J. Phys. Chem. Ref. Data, vol. 7, no. 3, pp. 959-1177, 1978.

[27] J. Mateu, C. Collado, N. D. Orloff, J. C. Booth, E. Rocas, A. Padilla, and J. M. O'Callaghan, "Third-order intermodulation distortion and harmonic generation in mismatched weakly nonlinear transmission lines," IEEE Trans. Microw. Theory Tech., vol. 57, no. 1, pp. 10-18, Jan. 2009.

[28] J. Mateu, J. C. Booth, and S. A. Schima, "Frequency tuning and spurious signal generation at microwave frequencies in ferroelectric SrTiO3 thin-film transmission lines," IEEE Trans. Microw. Theory Tech., vol. 55, no. 2, pp. 391-396, Feb. 2007.

[29] W. T. Weeks, L. L. Wu, M. F. McAllister, and A. Singh, "Resistive and inductive skin effect in rectangular conductors," IBM J. Res. Develop., vol. 23, no. 6, pp. 652-660, Nov. 1979.

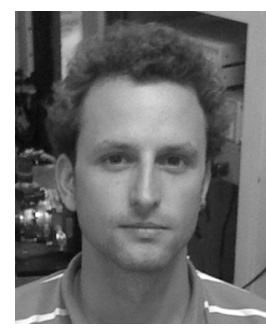

Eduard Rocas (S'07) received the Telecommunication Engineering degree from the Universitat Politècnica de Catalunya (UPC), Barcelona, Spain, in 2005, where his final project was associated with the creation of the Intelligent Communications and Avionics for Robust Unmanned Aerial Systems (ICARUS) Research Group, and is currently working toward the Ph.D. dgree at UPC.

From September 2005 to July 2006, he was involved in the simulation and modeling of advanced SONARs with the Computer Vision and Robotics Group (VICOROB), University of Girona, as an Formación del Profesorado Universitario (FPU) Grant Holder. Since November 2006, he has been with UPC as a doctoral student (Formación de Personal Investigador (FPI) Grant Holder), where his research is focused on new materials and structures for novel RF/microwave (MW) devices. Since January 2009, he has been a Guest Researcher with the National Institute of Standards and Technology (NIST), Boulder, CO. 


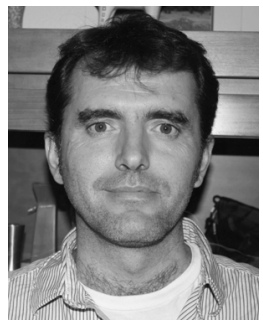

Carlos Collado (A'02-M'03-SM'10) received the Telecommunication Engineering degree, Ph.D. degree, and M.S. degree in biomedical engineering degree from the Universitat Politècnica de Catalunya (UPC), Barcelona, Spain, in 1995, 2001, and 2002, respectively.

In 1998, he joined the faculty of UPC, and became an Associate Professor in 2005. From November 2005 to January 2008, he was Vice-Dean of the Technical School of Castelldefels (EPSC), UPC, where he was responsible of the telecommunication and aeronautic engineering degrees. In 2004, he was a Visiting Researcher with the University of California at Irvine. From 2009 to 2010, he was a Guest Researcher with the National Institute of Standards and Technology (NIST), Boulder, $\mathrm{CO}$. His primary research interests include microwave devices and systems with focus on nonlinearities of passive devices.

Dr. Collado was the recipient of a 2001 prize for the best doctoral thesis in electronics and telecommunications from UPC.

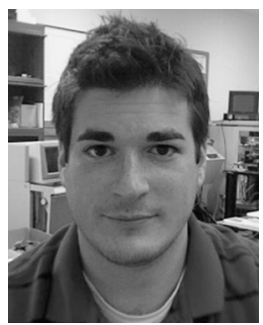

Nathan D. Orloff was born in Columbia, SC, on August 10, 1981. He received the B.S. degree in physics (with high honors) and Ph.D. degree from the University of Maryland at College Park, in 2004 and 2010, respectively. His doctoral research focused on the development of a broadband on-wafer calibration and characterization approach that has been applied to number of novel material systems.

$\mathrm{He}$ is interested in novel dielectric characterization techniques, complex-nonlinear device modeling, on-wafer measurement, and the integration of microelectronics and microfluidics.

Dr. Orloff was the recipient of the 2004 Martin Monroe Undergraduate Research Award, the 2006 CMPS Award for Excellence for Teaching Assistants, the 2010 Michael J. Pelczar Award for Excellence in Graduate Study, and an honorable mention for the 2010 Block Award for Best Poster Presentation from the Aspen Center for Physics.

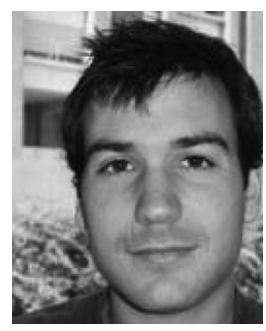

Jordi Mateu (M'03-SM'10) received the Telecommunication Engineering and Ph.D. degrees from the Universitat Politècnica de Catalunya (UPC), Barcelona, Spain, in 1999 and 2003, respectively.

In October 2006, he joined the Signal Theory and Communications Department, UPC, initially as a Ramon-y-Cajal Research Fellow, where since March 2009, he has been an Associate Professor. Since 2007 he has also been an Associate Researcher with the Centre Tecnològic de Telecomunicacions de Catalunya (CTTC), Castelldefels, Spain. From May to August 2001, he was a Visiting Researcher with Superconductor Technologies Inc., Santa Barbara CA. From October 2002 to August 2005, he was a Research Associate with CTTC. Since September 2004, he has held several guest researcher appointments with the National Institute of Standards and Technology (NIST), Boulder, CO, where he was a Fulbright Research Fellow from September 2005 to October 2006. In July 2006, he was a Visiting Researcher with the Lincoln Laboratory, Massachusetts Institute of Technology (MIT). From September 2003 to August 2005, he was a Part-Time Assistant Professor with the Universitat Autònoma de Barcelona. In Summer 1999, following graduation, he was a Trainee Engineer with the Investment Technology Department, Gillette, Isleworth, U.K. He has authored or co-authored over 40 papers in international journal, over 55 contributions in international conferences, and three book chapters. He holds two patents. He has collaborated and led several research projects for national and international public and private organizations and companies. He is reviewer of several journals and international conferences. His primary interests include microwave devices and system and characterization and modeling of new electronic materials including ferroelectrics, magnetoelectric, superconductors, and acoustic devices. His recent research interests also include the synthesis, design, and development of novel microwave filtering structures.

Dr. Mateu was the recipient of the 2004 Prize for the best doctoral thesis in Fundamental and Basic Technologies for Information and Communications by the Colegio Oficial de Ingenieros de Telecomunicación (COIT) and Asociación Española de Ingenieros de Telecomunicación (AEIT). He was also the recipient of a Fulbright Research Fellowship, an Occasional Lecturer Award for visiting MIT, and a Ramón y Cajal Contract.

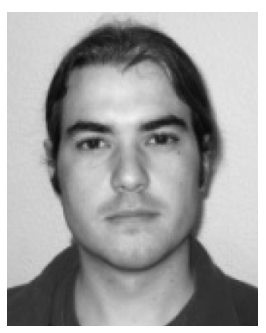

Alberto Padilla was born in Barcelona, Spain, in 1984. He received the Telecommunication Engineering degree from the Technical University of Catalonia (UPC), Barcelona, Spain, in 2008. His final project concerned the mitigation of the nonlinear behavior of high-temperature superconducting planar devices.

Since March 2008, he has been with the Department of Signal Theory and Communications, UPC. He is currently a Trainee-Stagiaire Researcher with the European Space Agency (ESA)-European Space Research and Technology Centre (ESTEC), where his focus is focused on the synthesis and design of a new class of receiver filters for satellite communications.

Mr. Padilla was the recipient of a Ph.D. grant (FPU) from the Spanish Ministry of Education in January 2009.

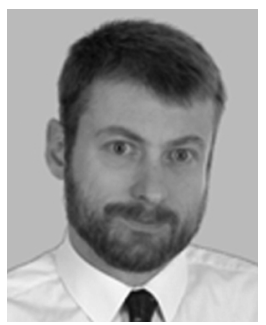

Juan M. O'Callaghan (SM'01) received the Telecommunication Engineering degree from the Universitat Politècnica de Catalunya (UPC), Barcelona, Spain, in 1987, and the Ph.D. degree from the University of Wisconsin-Madison, in 1993.

In 2003, he became a Full Professor with UPC. In 1989, he was with the Systems Research Center, Honeywell, Bloomington, $\mathrm{MN}$, where he was involved with noise measurement methods for field-effect transistors (FETs) at $\mathrm{Ka}$-band. In 1993 , he became a tenure-track faculty member with UPC. From 2003 to 2006, he was a Manager for MERIT, a consortium of European universities delivering a joint Master's program in Information Technologies within the Erasmus Mundus Program (see www.meritmaster.org). From 2006 to 2009, he was Vice-Dean of academic affairs with Telecom BCN, the telecommunication engineering school of UPC (see www.telecombcn.upc.edu). $\mathrm{He}$ has coauthored over 55 papers in peer-reviewed international journals and 90 contributions to conference proceedings. He holds four patents. His research interests include microwave devices and materials and microwave photonics. $\mathrm{He}$ has been involved with noise characterization, large-signal properties of GaAs FETs, and advanced microwave materials such as superconductors and ferroelectrics.

Dr. O'Callaghan was the recipient of the 2001 prize to the most outstanding research project from the Catalan Association of Telecommunication Engineers (ACET). In 2004, one of the theses he supervised received the prize for the best doctoral thesis in fundamental and basic technologies for information and communications by the Colegio Oficial de Ingenieros de Telecomunicación (COIT) and Asociación Española de Ingenieros de Telecomunicación (AEIT).

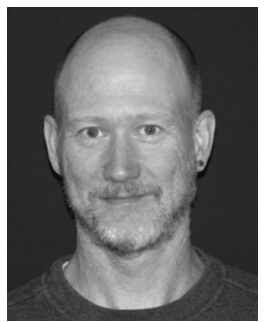

James C. Booth received the B.A. degree in physics from the University of Virginia, Charlottesville, in 1989, and the Ph.D. degree in physics from the University of Maryland at College Park, in 1996. His doctoral dissertation concerned novel measurements of the frequency dependent microwave surface impedance of cuprate thin film superconductors.

Since 1996, he has been a Physicist with the National Institute of Standards and Technology (NIST), Boulder, CO, initially as aNational Research Council (NRC) Postdoctoral Research Associate (1996-1998) and currently as the leader of the High Frequency Devices and Characteristics Project. His research with NIST is focused on exploring the microwave properties of new electronic materials and devices, including ferroelectric, magneto-electric, and superconducting thin films, as well as the development of experimental platforms integrating microfluidic and microelectronic components for RF and microwave frequency characterization of liquid and biological samples. 